\title{
Severe Jaccoud's arthropathy in systemic lupus erythematosus
}

\author{
Mittermayer B. Santiago ${ }^{1,2} \cdot$ Verena Galvão $^{1} \cdot$ Daniel Sá Ribeiro $^{1} \cdot$ Willer D. Santos ${ }^{1}$ • \\ Priscila R. da Hora ${ }^{1}$ Anna Paula Mota ${ }^{1} \cdot$ Emanuela Pimenta $^{1} \cdot$ Isabela Oliveira $^{3}$. \\ Ajax M. Atta ${ }^{3}$ Mitermayer G. Reis ${ }^{4}$ Eliana A. G. Reis ${ }^{4}$ Carolina Lins ${ }^{1}$
}

Received: 15 June 2015 / Accepted: 18 August 2015 / Published online: 27 August 2015

(C) Springer-Verlag Berlin Heidelberg 2015

\begin{abstract}
Jaccoud's arthropathy (JA) is a clinical situation nowadays present mostly in systemic lupus erythematosus (SLE). It is characterized by the presence of joint deformities such as "swan neck," ulnar deviation and "Z-thumb" resembling rheumatoid arthritis (RA) but that are passively correctable and without bone erosion on plain radiographs. From our cohort of SLE patients with JA, we selected a subgroup with a more severe form of this arthropathy and looked at their clinical and laboratory profile as well as studied the magnetic resonance imaging (MRI) findings or ultrasound (US) obtained from the hand with most evident deformities. Seven SLE patients with a severe form of JA were identified. All seven patients have "swan neck," ulnar deviation and "Z-thumb" deformities. Two out of seven had "mutilans-type JA" and four had fixed deformities in the metacarpophalangeal (MCP) joints. The MRI of the hand with more evident deformity clinically performed in six cases and US performed in one case showed mild synovitis in five and moderate synovitis in two patients, mild flexor tenosynovitis in six and severe tenosynovitis in one. Only two small bone erosions were observed in the second and third MCP joints of one patient with moderate synovitis.
\end{abstract}

Mittermayer B. Santiago

mbsantiago2014@gmail.com

1 Escola Bahiana de Medicina e Saúde Pública, Av. Dom João VI, 275, Brotas, Salvador, BA 40290-000, Brazil

2 Serviços Especializados em Reumatologia da Bahia, Rua Conde Filho, 117, Graça, Salvador, BA 40150-150, Brazil

3 Departamento de Análises Clínicas e Toxicológicas, Faculdade de Farmácia, Universidade Federal da Bahia, Salvador, BA 40000-000, Brazil

4 Laboratório de Biologia Molecular-Fundação Gonçalo Muniz (Fiocruz), Salvador, BA 40000-000, Brazil
Severe JA compromises the functional capacity of the joints and imposes the risk of misdiagnosis of RA. With the improvement of the survival rate of SLE and the lack of specific prophylactic or therapeutical measures for JA, it is reasonable to assume that more and more cases of severe $\mathrm{JA}$ are going to be identified.

Keywords Jaccoud's arthropathy $\cdot$ Systemic lupus erythematosus $\cdot$ Deforming arthropathy

\section{Introduction}

Jaccoud's arthropathy (JA) is characterized by joint deformities similar to those classically seen in rheumatoid arthritis (RA) such as "swan neck," ulnar deviation and "Z-thumb," but with a "reversible" pattern and with no erosions on plain radiograph. Initially, it was described in patients with rheumatic fever (RF), but presently it has been seen mainly in systemic lupus erythematosus (SLE) with a prevalence of about $5 \%$ [1].

Although the term "reversible" has been widely used to define the deformities seen in JA, it seems to be inappropriate because these deformities last forever. Moreover, although in the majority of the cases the "correctable" nature of the arthropathy can be easily demonstrated, sometimes the joints have limitation of movement-even in the absence of bone erosions-causing significant morbidity and limitation of the quality of life of the patients [2]. This fixed character is claimed to be secondary to soft tissue fibrosis, disuse of the involved joints and muscle hypotrophy [1].

In the present study, we analyze the clinical, laboratory and imaging characteristics of a group of SLE patients with severe JA, a condition distinct from the erosive form of the 
deforming arthropathy observed in the association of SLE and RA (rhupus) [3].

\section{Materials and methods}

The initial inclusion criteria for the present study were diagnosis of SLE based on the American College of Rheumatology (ACR) revised criteria [4] followed at our outpatient clinic and the presence of JA as defined by the following findings [5]: (1) typical joint deformities such as "swan neck," thumb subluxation, ulnar deviation, "boutonniere," genu recurvatum, hallux valgus and flat feet; (2) presence or history of articular inflammation in the deformed joints; (3) absence of similar deformities in other healthy members of the same family; (4) no erosion on plain radiographs regardless of its presence on magnetic resonance imaging (MRI) or highperformance ultrasound (US) examination. After selecting these patients with SLE and JA, a second selection was performed to identify only those with a more "severe subtype" of JA arbitrarily defined as the presence of deformities of at least three types with or without fixed deformities.

For those patients who fulfilled these criteria, complete clinical evaluation was performed. Data were then obtained from interviewing, physical examination, reviewing patient records, blood samples taken for laboratory tests and MRI or US study of the most deformed hand. All the patients signed an informed consent form, and the ethical committee of our institution approved the study.

\section{Results}

From our cohort of 1052 SLE patients, 53 were identified as having JA, and from this group, seven (including six women and one man) were defined as "severe JA." The age ranged from 44 to 79 years (mean $54.5 \pm 11.9$ years), and the age at the time of the diagnosis ranged from 16 to 31 years (mean $22 \pm 5.3$ years). All patients had arthritis as part of the initial findings of the disease. The most common joint deformities were the "swan neck" type, ulnar deviation and Z-thumb observed in all seven patients. Four patients had "fixed" or no correctable deformities in the metacarpophalangeal (MCP) joints. A clear subluxation was observed in some joints. The joint deformities were very impressive in all patients assuming a "mutilans-type JA" character in two of them. The clinical features of one of these two patients have been published elsewhere [6].

Leukopenia was detected in six out of seven patients. A low-titer rheumatoid factor was observed in four patients, and borderline anti-cyclic citrullinated peptide
(CCP) antibody positivity was detected in only one patient. Interestingly, all seven patients had present or past anti-DNA antibodies positivity, but only three had a history of nephritis defined as proteinuria higher than $1 \mathrm{~g} /$ day with or without increased serum creatinine. Present or past treatment regimen included steroids, hydroxychloroquine, cyclophosphamide, azathioprine and methotrexate. As part of the inclusion criteria, a plain radiograph of the hands did not show any erosion although subluxation was a very common finding. MRI (six patients) or US (one patient) of the hand with more evident deformity clinically showed mild synovitis in five and moderate synovitis in two patients, mild flexor tenosynovitis in six and severe tenosynovitis in one. Only two small bone erosions were observed in the second and third MCP joints in one patient who also presented moderate synovitis. Table 1 presents the main clinical, laboratory and imaging findings of the seven patients, while Fig. 1 shows the illustration of some hand deformities seen in the present series.

\section{Discussion}

Although described more than a century ago, JA has received little attention in clinical practice. Nowadays, its presence in RF has been described rarely, first because RF is becoming infrequent and second because the joint deformities are regarded of secondary importance in those patients with valvular rheumatic lesions and candidates for surgical procedures. The prevalence of JA is $4.2 \%$ in this scenario [7]. Likewise, even in SLE where the JA has been most identified in the present days, this arthropathy has been seen as of low relevance in the context of the disease. The present series of severe joint deformities in JA draws one's attention to the fact that in some cases it cannot be considered as a benign condition. Although other series of deforming arthropathy in SLE patients have been published, they did not specify how many patients had the severe form of JA $[8,9]$.

JA can bring an important functional limitation of the joints, sometimes in a "non-reversible" or fixed manner resembling those deformities seen in RA, although without bone erosion on plain radiographs as seen in case four cases of the present series. It certainly has a negative cosmetic and functional impact and represents a significant compromise in the quality of life for these patients. Of note, one of our patients used to be a taxi driver, but he was forced to abandon his profession due to the joint deformities [10]. It should be noted that the above-mentioned "irreversible" pattern of JA can be easily confounded with RA. 


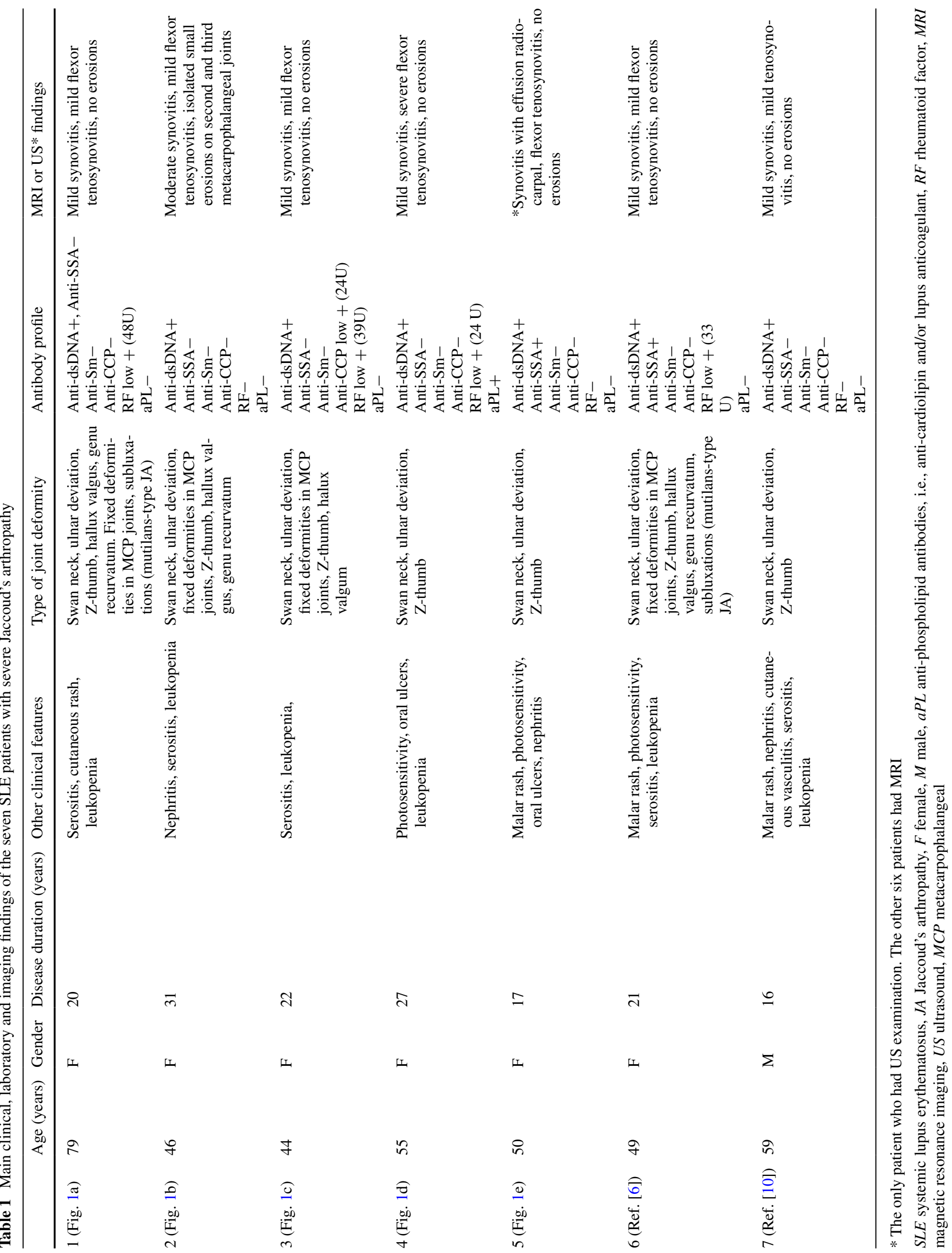




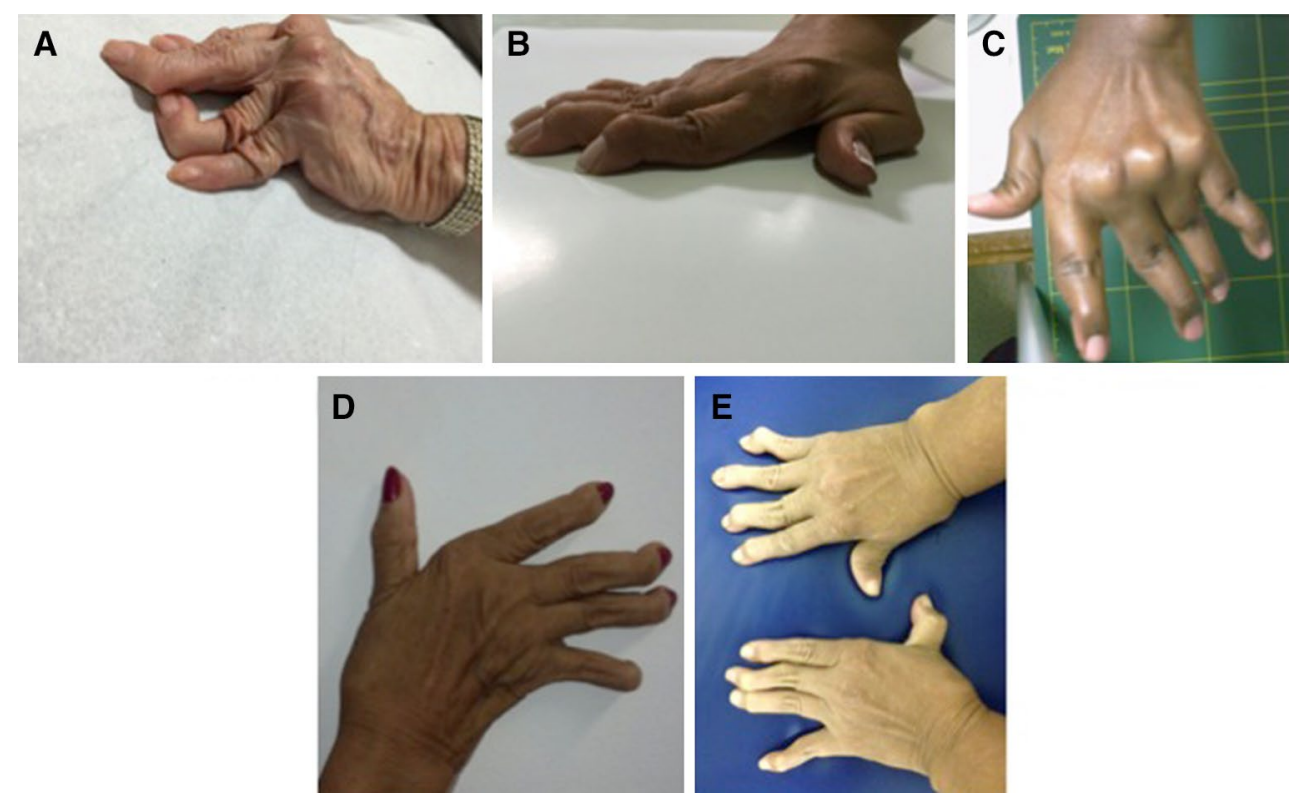

Fig. 1 Illustration of hand deformities seen in five out seven SLE patients with Jaccoud's arthropathy included in the present series (a-e). The two other cases have been published elsewhere $[6,10]$

Another negative aspect linked to JA is the observation that it is a risk factor for tendon rupture. In one systematic review of the literature, in 55 SLE patients with tendon rupture, 16 (29\%) had JA [11]. This complication is probably related to an inflammatory process of the tendon sheath and consequent weakness of the tendon itself. Theoretically, in more severe forms of JA with loss of biomechanical properties of the joints, more tendon rupture is expected to occur.

The etiopathogenic mechanisms for JA are not known. There are a few published studies trying to associate JA with the presence of some antibodies. Thus, anti-SSA/Ro, $\mathrm{RF}$ and anti-cardiolipin antibodies are among the candidates. Previously, we found an association between the presence of anti-dsDNA antibodies and JA [12], and in the present series of severe JA, all seven patients had such antibodies in at least one moment of their disease. Curiously, only three had history of lupus nephritis. However, the role, if any, for these antibodies in JA needs to be proved. Another important observation from our findings is that even with more aggressive clinical picture, only one of the patients had low titer of anti-CCP antibodies making these antibodies ineligible candidates to participate in the pathogenesis of this arthropathy.

Another example of how JA has been neglected is the lack of studies regarding its management. Physical therapy, orthesis and corticosteroids are described as therapeutical options based on expert opinion. A few studies have been published on the surgical procedures for JA [13-15], but there is no certainty about the best modality, when to indicate and the long-term follow-up. From those studies, one can identify other examples of "severe JA," some of them with fixed deformities and aggressive enough to be candidates for the surgical procedures. Recently, we demonstrated that the level of interleukin 6 was higher in SLE patients with JA as compared to those without such complication [16]. Although preliminary, these findings emphasize the necessity for new studies with a larger number of patients and perhaps evaluating the potential therapeutical benefit for blocking this molecule in SLE before the development of joints deformities as seen in JA. Curiously, recently published studies have demonstrated the efficacy of the utilization of anti-TNF-alpha blockers in SLE patients with severe arthritis $[17,18]$. Although regarded as potential inductor of the autoimmune processes such as lupus, demyelinating disorders and psoriasis, this treatment modality, in selected cases of SLE, may be useful. To the best of our knowledge, there is no case of severe JA treated with such agents.

With the improvement of the survival rate of SLE and the lack of specific prophylactic or therapeutical measures for JA, it is reasonable to assume that more and more cases of "severe JA" are going to be identified.

Acknowledgments Mittermayer B. Santiago, Ajax M. Atta and Mitermayer G. Reis are currently receiving a scholarship from Conselho Nacional de Desenvolvimento Científico e Tecnológico (CNPq).

\section{Compliance with ethical standards}

Conflict of interest The authors have no conflict of interest that is directly relevant to the content of this manuscript. 


\section{References}

1. Santiago MB (2011) Miscellaneous non-inflammatory musculoskeletal conditions. Jaccoud's arthropathy. Best Pract Res Clin Rheumatol 25(5):715-725 (Epub 2011/12/07)

2. Santiago MB, Galvao V (2008) Jaccoud arthropathy in systemic lupus erythematosus: analysis of clinical characteristics and review of the literature. Medicine (Baltimore) 87(1):37-44 (Epub 2008/01/22)

3. van Vugt RM, Derksen RH, Kater L, Bijlsma JW (1998) Deforming arthropathy or lupus and rhupus hands in systemic lupus erythematosus. Ann Rheum Dis 57(9):540-544 (Epub 1998/12/16)

4. Hochberg MC (1997) Updating the American College of Rheumatology revised criteria for the classification of systemic lupus erythematosus. Arthritis Rheum 40(9): 1725 (Epub 1997/10/27)

5. Santiago MB (2013) Jaccoud's arthropathy: proper classification criteria and treatment are still needed. Rheumatol Int 33(11):2953-2954 (Epub 2012/09/06)

6. Santiago MB, Machicado V, Ribeiro DS (2015) "Mutilanstype" Jaccoud Arthropathy. J Rheumatol 42(4):725-726 (Epub 2015/04/04)

7. Moro C, Castro C, Lanza MG, Font de Mora A, Cosio FG, Ruigomez L et al (1978) Jaccoud's arthropathy in patients with chronic rheumatic valvular heart disease. Eur J Cardiol 6(6):459-468 (Epub 1978/02/01)

8. Alarcon-Segovia D, Abud-Mendoza C, Diaz-Jouanen E, Iglesias A, De los Reyes V, Hernandez-Ortiz J (1988) Deforming arthropathy of the hands in systemic lupus erythematosus. J Rheumatol 15(1):65-69 (Epub 1988/01/01)

9. Bleifeld CJ, Inglis AE (1974) The hand in systemic lupus erythematosus. J Bone Joint Surg Am Vol 56(6):1207-1215 (Epub 1974/09/01)
10. Santiago M, Machicado V (2015) Images in clinical medicine. Jaccoud's Arthropathy. N Engl J Med 373(1):e1 (Epub 2015/07/02)

11. Alves EM, Macieira JC, Borba E, Chiuchetta FA, Santiago MB (2010) Spontaneous tendon rupture in systemic lupus erythematosus: association with Jaccoud's arthropathy. Lupus 19(3):247254 (Epub 2009/12/03)

12. Galvao V, Atta AM, Sousa Atta ML, Motta M, Dourado S, Grimaldi L et al (2009) Profile of autoantibodies in Jaccoud's arthropathy. Joint Bone Spine 76(4):356-360 (Epub 2009/03/13)

13. Dray GJ, Millender LH, Nalebuff EA, Philips C (1981) The surgical treatment of hand deformities in systemic lupus erythematosis. J Hand Surg Am 6(4):339-345 (Epub 1981/07/01)

14. Evans JA, Hastings DE, Urowitz MB (1977) The fixed lupus hand deformity and its surgical correction. $\mathrm{J}$ Rheumatol 4(2):170-175

15. Schumacher HR, Zweiman B, Bora FW Jr (1976) Corrective surgery for the deforming hand arthropathy of systemic lupus erythematosus. Clin Orthop Relat Res 117:292-295 (Epub 1976/06/11)

16. Atta AM, Oliveira RC, Oliveira IS, Menezes MP, Santos TP, Sousa Atta ML et al (2015) Higher level of IL-6 in Jaccoud's arthropathy secondary to systemic lupus erythematosus: a perspective for its treatment? Rheumatol Int 35(1):167-170 (Epub 2014/06/19)

17. Mosca M, Tani C, Filice ME, Carli L, Delle Sedie A, Vagnani S et al (2015) TNF-alpha inhibitors in systemic lupus erythematosus. A case report and a systematic literature review. Mod Rheumatol 25(4):642-645 (Epub 2013/11/21)

18. Cortes-Hernandez J, Egri N, Vilardell-Tarres M, Ordi-Ros J (2015) Etanercept in refractory lupus arthritis: an observational study. Semin Arthritis Rheum 44(6):672-679 (Epub 2015/02/26) 